\title{
Enhanced Indoor Navigation Using Fusion of IMU and RGB-D Camera
}

\author{
W.N. Chai \\ Center for Sensor Systems (ZESS) \\ University of Siegen \\ Germany
}

\author{
C. Chen \\ Institute of Automatic Control Engineering (RST) \\ University of Siegen \\ Germany
}

\author{
E. Edwan \\ Chair for Circuit Design and Network \\ Dresden University of Technology \\ Germany
}

\begin{abstract}
Accurate indoor navigation, especially precise attitude estimation is a challenge topic. Unlike the rate gyroscope in an IMU, the camera based visual-gyro does not suffer from drift errors. In order to overcome the drawbacks of the standalone systems, an INS/visual-gyro integration using direction cosine matrix (DCM) models is presented. Compared to the conventional Euler angle models, the usage of DCM can provide linear system models and avoided singularity problems. Using iterative closest point (ICP) algorithm, the depth measurement from a RGB-D camera can be converted to positioning information and further added to the integrated navigation system. To show the performance of the presented system, one field experiment is carried out with a mobile robot and the numerical results are shown.
\end{abstract}

Keywords-Inertial measurement unit (IMU); Visual gyroscope; Direction cosine Matrix (DCM); Iterative closest point (ICP); Kalman filtering

\section{INTRODUCTION}

Precise attitude estimation is a challenge topic for indoor navigation. Microelectromechanical systems (MEMS) based inertial measurement units (IMUs) are widely used in navigation applications. The attitude determination using standalone IMU suffers from drift error propagation because of gyro biases. The magnetometer is affected by magnetic disturbances, which limit the use of magnetometer for attitude determination in indoor applications. In this case, the camerabased direction assistance was proposed to improve the heading estimation.

In this work, an INS/visual-gyro integration using direction cosine matrix (DCM) based models is presented. The INS/visual-gyro integration using the DCM attitude representation avoids the singularity problem that occurs with Euler angle representations. Furthermore, a linear (or pseudolinear if augmenting the gyro bias) process model for the DCM update with respect to its parameters is formulated. Moreover, a linear observation model is derived for visual-gyro triad with respect to the DCM elements.

The advent of RGB-D sensors such as Microsoft Kinect has resulted in great progress due to its low cost and high output frame rate. By using a Kinect sensor, many works for standalone camera position tracking have been introduced. RGBD odometry is introduced recently as a real time application [1]. By using iterative closest point (ICP) algorithm [2], Kinect fusion (KinFu) [3] has demonstrated great results for indoor environment reconstruction. However, both KinFu and RGB-D algorithm works pair-wisely. Errors from each registration pair can be accumulated and results in positioning and attitude drifts.

Making use of the complementary nature of INS and vision/range based systems; the combination of them is expected to provide a synergetic solution. The integration method is attractive as it is a trend of the usage of $2 \mathrm{D} / 3 \mathrm{D}$ camera in navigation applications.

The rest of this paper is organized as follows. The second section introduces visual gyroscope using vanishing points. The vanishing point finding approach and the camera attitude determination method used in this work are described in this section. The third section presents INS/visual-gyro/ICP integration. In this section, DCM based INS/Visual-gyro system modelling and the system block diagram are given in detail. In the fifth section, one field experiment is described and the numerical results are presented. Finally, conclusions of this research are provided.

\section{VISUAL GYROSCOPE USING VANISHING POINTS}

The visual gyroscope technique makes use of computer vision algorithms to transform information found from images into the camera rotation. Unlike rate gyroscope in a MEMS based IMU, the visual gyroscope does not suffer from drift errors. Therefore, in this work, it is used to calibrate the IMU gyro output through INS/visual-gyro integration. The visual gyroscope employed here is based on tracking vanishing points in consecutive images.

The vanishing points are the points in an image where the lines parallel in the real world seem to intersect. The detected motion of the vanishing points can be converted into camera rotation. There are normally three useful vanishing points in an image which are central, vertical and horizontal vanishing points. They are intersects of the parallel lines along 3 
orthogonal axes. This algorithm is suitable for indoor environments, since the human made constructions are mainly composed of orthogonal structures and the parallel lines as well as the vanishing points can be detected.

Some steps are needed to calculate the locations of the vanishing points [4]. Firstly, the images taken in low-light environments are filtered out using Gaussian convolution filter [5]. Then edges of objects are located by tracing fast changes in pixels' brightness values using the canny edge detector and straight lines are separated from other edges using the Hough line detector [6]. The last step is finding the vanishing point locations in the image frame, which is using the random sample consensus (RANSAC) method [7]. The processing results of edge detection, parallel line detection and vanishing point finding are shown in Figure 1. The detected parallel lines along 3 orthogonal axes are marked with red, green, blue colors respectively. Only the central vanishing point is shown in the figure because the other two vanishing points are located outside the image scope.

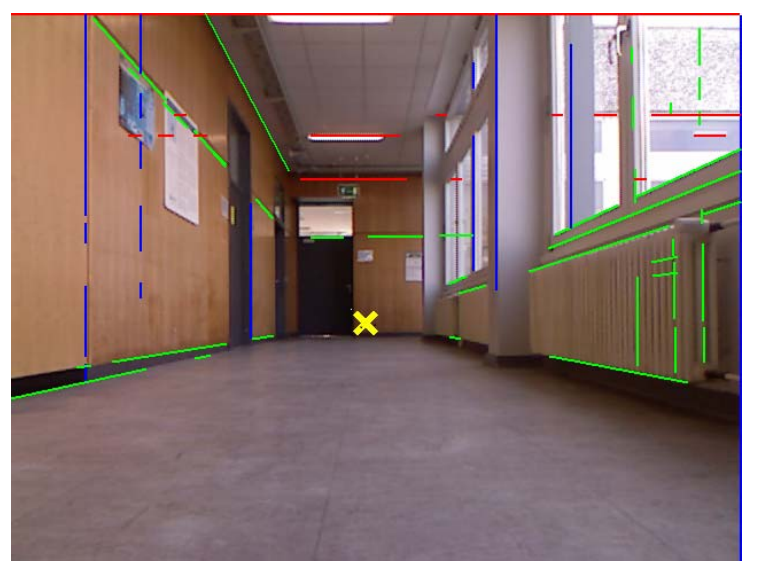

FIGURE I. EDGE DETECTION, LINE DETECTION AND VANISHING POINT FINDING.

After vanishing point is successfully found, the remaining task is to exact information of the attitude changes from the locations of the vanishing points. The rotation matrix of the camera may be resolved by using VPs and the calibration matrix of the camera, which is shown with equation: $\mathbf{V}=\mathbf{K} \mathbf{R}$ where $\mathbf{V}$ is the vanishing point location matrix, $\mathbf{R}$ is the calibration matrix and $\mathrm{K}$ is the camera rotation matrix. The calibration matrix $\mathbf{K}$ contains information of the intrinsic camera parameters, which includes the focal length and the principal point. They can be approximated using the values in the image file headers, yet their exact values are obtained by calibrating the camera. The matrix $\mathbf{R}$ is the three-dimensional rotation of the camera.

However, there are limitations of the visual-gyro application. For instance, the visual-gyro does not work when the vanishing point finding is not available, which happens when no vanishing point can be found or too many disturbing lines detected in the image. Moreover, since the high computational requirement, the frequency of visual-gyro output is relatively low and the performance for fast rotation estimation is limited.

\section{INS/VISUAL-GYRO/ICP INTEGRATION}

\section{A. DCM based INS/Visual-gyro System Modelling}

However, making use of the complementary nature of INS and visual gyroscope, the INS/visual-gyro integration is expected to yield a synergetic effect, which can overcome the limitations of both systems. In this work, DCM based system models are used for INS/visual-gyro integration to estimate the attitude.

The advantage of using a DCM based model is having a linear process model for the DCM update with respect to its parameters. However, augmenting the gyro bias vector makes the process model non-linear which can be handled using a pseudo-linear process model [8]. Another important benefit of using the DCM attitude representation is avoiding the singularity problem that occurs with Euler angle representation.

The strap-down INS mechanization regarding the DCM matrix is shown as the following equation [9]: $\dot{\mathbf{C}}_{\mathrm{b}}^{\mathrm{n}}=\mathbf{C}_{\mathrm{b}}^{\mathrm{n}} \boldsymbol{\omega}_{\mathrm{nb}} \times$, where $\mathbf{C}_{\mathrm{b}}^{\mathrm{n}}$ is the DCM rotation matrix that transforms the inertial measurements from the body frame to the local navigation frame and $\boldsymbol{\omega}_{\mathrm{nb}}$ is the turn rate of body frame with respect to the local navigation frame. The elements of the DCM are trigonometric functions of the Euler angles defined as

$$
\mathbf{C}_{\mathrm{b}}^{\mathrm{n}}=\left[\begin{array}{ccc}
\mathrm{C} \varphi \mathrm{C} \phi & \mathrm{C} \varphi \mathrm{S} \phi \mathrm{S} \theta-\mathrm{S} \varphi \mathrm{C} \theta & \mathrm{C} \varphi \mathrm{S} \phi \mathrm{C} \theta+\mathrm{S} \varphi \mathrm{S} \theta \\
\mathrm{S} \varphi \mathrm{C} \phi & \mathrm{S} \varphi \mathrm{S} \phi \mathrm{S} \theta+\mathrm{C} \varphi \mathrm{C} \theta & \mathrm{S} \varphi \mathrm{S} \phi \mathrm{C} \theta-\mathrm{C} \varphi \mathrm{S} \theta \\
-\mathrm{S} \phi & \mathrm{C} \phi \mathrm{S} \theta & \mathrm{C} \phi \mathrm{C} \theta
\end{array}\right]=\left[\begin{array}{ccc}
c_{11} & c_{12} & c_{13} \\
c_{21} & c_{22} & c_{23} \\
c_{31} & c_{32} & c_{33}
\end{array}\right]
$$

For the integration system, the state vector to be estimated is composed of the nine elements of the DCM and gyro triad bias vector, which is described as the following form:

$$
\begin{aligned}
& \mathbf{x}=\left[\begin{array}{llll}
\mathbf{c}_{1}^{T} & \mathbf{c}_{2}^{T} & \mathbf{c}_{3}^{T} & \mathbf{b}_{\text {gyro }}^{T}
\end{array}\right]^{T} \\
& \text { where } \\
& \mathbf{c}_{1}=\left[\begin{array}{lll}
c_{11} & c_{12} & c_{13}
\end{array}\right]^{T}, \mathbf{c}_{2}=\left[\begin{array}{lll}
c_{21} & c_{22} & c_{23}
\end{array}\right]^{T}, \mathbf{c}_{3}=\left[\begin{array}{lll}
c_{31} & c_{32} & c_{33}
\end{array}\right]^{T} \\
& \mathbf{b}_{\text {gyro }}^{T}=\left[\begin{array}{lll}
b_{\text {gyro }, x} & b_{\text {gyro, } y} & b_{\text {gyro, },}
\end{array}\right]^{T}
\end{aligned}
$$

The DCM propagation is given as $\mathbf{C}_{\mathrm{b}, k}^{\mathrm{n}}=\mathbf{C}_{\mathrm{b}, k-1}^{\mathrm{n}} \mathbf{A}_{k-1}$, where $\mathbf{A}_{k-1}=e^{\int_{k-1}^{t_{k}} \boldsymbol{\omega}_{\mathrm{ib}}^{\mathrm{b}} \times d t} \cdot \boldsymbol{\omega}_{\mathrm{ib}}^{\mathrm{b}}$ is rotation rate of the body frame [10]. Assuming that the angular velocity vector has little change during the update interval, we can approximate the angle rotation vector $\boldsymbol{\sigma}$ as $\boldsymbol{\sigma}=\int_{t_{k-1}}^{t_{k}} \boldsymbol{\omega}_{\mathrm{ib}}^{\mathrm{b}} d t, \boldsymbol{\sigma} \approx \boldsymbol{\omega}_{\mathrm{ib}}^{\mathrm{b}} \Delta t$. In this case, $\mathbf{A}_{k-1}$ can be expanded and then reformulated with a second order approximation as:

$$
\mathbf{A}_{k-1} \approx\left[\begin{array}{ccc}
1-\frac{\left(\sigma_{y}^{2}+\sigma_{z}^{2}\right)}{2} & \frac{\sigma_{x} \sigma_{y}}{2}-\sigma_{z} & \frac{\sigma_{x} \sigma_{z}}{2}+\sigma_{y} \\
\frac{\sigma_{x} \sigma_{y}}{2}+\sigma_{z} & 1-\frac{\left(\sigma_{x}^{2}+\sigma_{z}^{2}\right)}{2} & \frac{\sigma_{y} \sigma_{z}}{2}-\sigma_{x} \\
\frac{\sigma_{x} \sigma_{z}}{2}-\sigma_{y} & \frac{\sigma_{y} \sigma_{z}}{2}+\sigma_{x} & 1-\frac{\left(\sigma_{x}^{2}+\sigma_{y}^{2}\right)}{2}
\end{array}\right]
$$

By ignoring the quadratic terms of gyro bias and noise, A can be approximated and written in terms of the exact matrix $\tilde{\mathrm{A}}$ as $\mathbf{A}_{k-1} \approx \tilde{\mathbf{A}}_{k-1}-\left[\mathbf{b}_{\text {gyro }} \Delta t\right] \times$. 
The system process model can be derived as

$$
\begin{aligned}
\mathbf{x}_{k} & =\mathbf{F}_{k-1} \mathbf{x}_{k-1} \\
\mathbf{F}_{k-1} & =\left[\begin{array}{cccc}
\tilde{\mathbf{A}}_{k-1}^{T} & \mathbf{0}_{3 \times 3} & \mathbf{0}_{3 \times 3} & -\Delta t \mathbf{x}_{1: 3} \times \\
\mathbf{0}_{3 \times 3} & \tilde{\mathbf{A}}_{k-1}^{T} & \mathbf{0}_{3 \times 3} & -\Delta t \mathbf{x}_{4: 6} \times \\
\mathbf{0}_{3 \times 3} & \mathbf{0}_{3 \times 3} & \tilde{\mathbf{A}}_{k-1}^{T} & -\Delta t \mathbf{x}_{7: 9} \times \\
\mathbf{0}_{3 \times 3} & \mathbf{0}_{3 \times 3} & \mathbf{0}_{3 \times 3} & \mathbf{I}_{3 \times 3}
\end{array}\right]
\end{aligned}
$$

With system alignment between INS and visual-gyro, the DCM elements are linear related to the camera rotation matrix

$\mathbf{R}$. The Kalman filter is employed in this work for estimating the attitude and gyro-bias which is included in the state vector.

\section{B. System Block Diagram of INS/Visual-gyro/ICP Integration}

Using ICP algorithm, the depth measurement from a RGB$\mathrm{D}$ camera can be converted to localization information and further be added to the integrated system for position update.

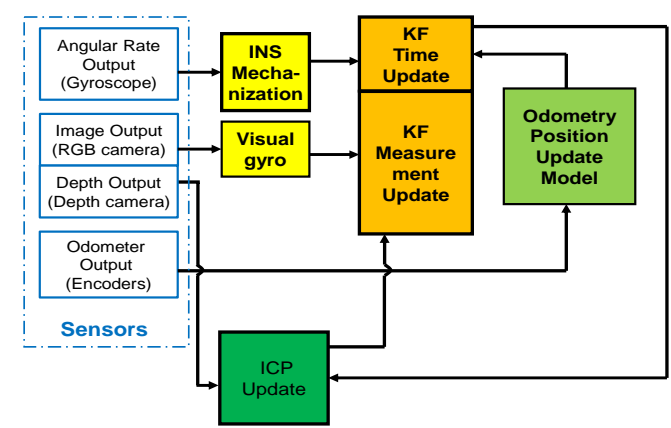

FIGURE II. INTEGRATED SYSTEM FOR MOBILE ROBOT NAVIGATION

Figure 2 shows the system block diagram of INS/Visualgyro/ICP integration for mobile robot navigation.

\section{FIELD EXPERIMENT}

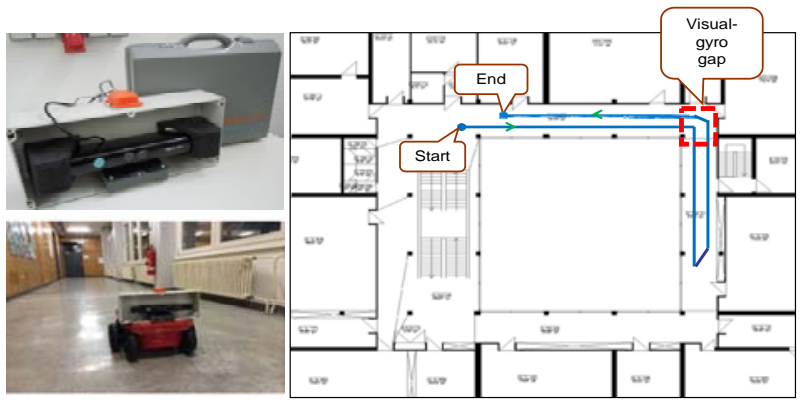

FIGURE III. EXPERIMENTAL HARDWARE AND SCENARIO.

One field experiment is contacted. The core experimental sensors are shown in Figure 3. The inertial sensor used here is MEMS Xsens MTi. The visual sensor employed here is Microsoft Kinect sensor, which can provide the synchronized RGB and D measurement. The Kurt2 mobile robot is used as the test platform. The experiment is contacted in the corridor of RST institute in University of Siegen. The trajectory of the mobile robot is shown with blue curve. The red marked part is visual-gyro gap area, where the visual-gyro is not available because of the vanishing point finding failures.

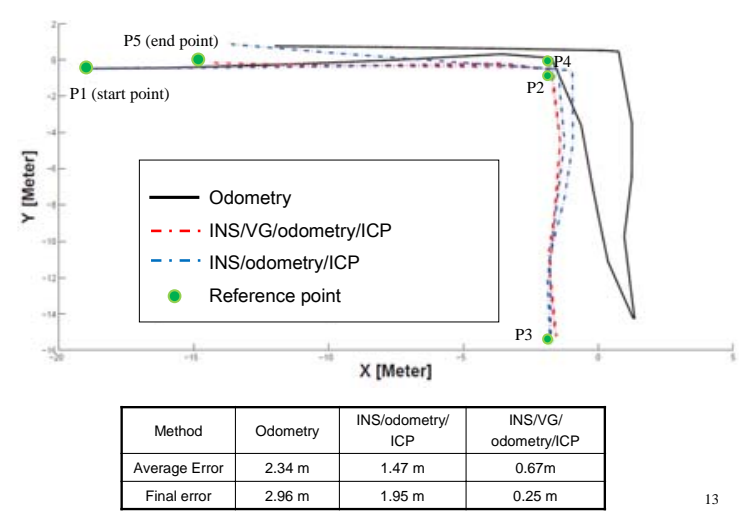

FIGURE IV. ROBOT TRAJECTORY ESTIMATION RESULT.

The trajectory estimation result is shown in Figure 3. The five green dots show the reference points in the true trajectory. The average position error and final error (distance to the end point) are shown in the table.

\section{CONCLUSION}

This paper explores the camera based visual gyroscope algorithm. A DCM based INS/visual-gyro integration is presented. In order to avoided singularity problems and obtain a linear or pseudo-linear system, the system modelling using DCM has been done. The INS/visual-gyro/ICP integration is employed for mobile robot navigation. One indoor experiment is carried out. The results show that the presented integration can bridge the visual-gyro gaps. It can also outperform the standalone odometry and INS/odometry integration in trajectory estimation.

\section{REFERENCE}

[1] C. Kerl, J. Sturm, D. Cremers, "Robust Odometry Estimation for RGB-D Cameras”, In Proc. Of the IEEE Int. Conf. on Robotics and Automation (ICRA), 2013

[2] P. Bsel and N. McKay. "A Method for registration of 3-D shapes”, IEEE Trans. Pattern Analysis and Machine Intelligence, 1992

[3] R. Newcombe, A. Davison, S. Izadi, etc. "KinectFusion: Real-time dense surface mapping and tracking”, 2011 10th IEEE International Symposium on Mixed and Augmented Reality (ISMAR), 2011

[4] C. Kessler, C. Ascher, N. Frietsch, M. Weinman, and G. Trommer, "Vision-based attitude estimation for indoor navigation using vanishing points and lines", PLANS, 2010

[5] D. Forsyth and J. Ponce, "Computer vision: a modern approach," Prentice Hall, 2003

[6] J. Canny, “A computational approach to edge detection”, IEEE Transactions on Pattern Analysis and Machine Intelligence, 1986

[7] M. A. Fischler, R. C. Bolles, "Random Sample Consensus: A Paradigm for Model Fitting with Applications to Image Analysis and Automated Cartography”, in: Communications of the Association for Computing Machinery 24, 1981

[8] D. Choukroun, "Novel Methods for Attitude Determination Using Vector Observations,” The Technion, PhD Dissertation, 2003

[9] Titterton, D. H. and Weston, J. L., "Strapdown Inertial Navigation Technology," 2nd ed, The American Institute of Aeronautics and Astronautics and The Institution of Electrical Engineers, 2004

[10] E. Edwan, J. Zhou, J. Zhang, and O. Loffeld, "A New Loosely Coupled DCM Based GPS/INS Integration Method,” Navigation: Journal of the Institute of Navigation, Vol 59. No. 2, 2012 